\title{
Higher-order derivative of intersection local time for two independent fractional Brownian motions *
}

\author{
Jingjun $\mathrm{Guo}^{a, \dagger}$ Yaozhong $\mathrm{Hu}^{b}$, Yanping Xiao ${ }^{c}$ \\ a.School of Statistics, Lanzhou University of Finance and Economics, \\ Lanzhou, GS 730020,P.R.China \\ b.Department of Mathematics, University of Kansas, \\ Lawrence, KS 66045, USA \\ c.School of Mathematics and Computer Science, \\ Northwest University for Nationalities, Lanzhou, GS 730000, P.R. China
}

\begin{abstract}
In this article, we obtain sharp conditions for the existence of the high order derivatives ( $k$-th order) of intersection local time $\widehat{\alpha}^{(k)}(0)$ of two independent d-dimensional fractional Brownian motions $B_{t}^{H_{1}}$ and $\widetilde{B}_{s}^{H_{2}}$ with Hurst parameters $H_{1}$ and $H_{2}$, respectively. We also study their exponential integrability.
\end{abstract}

Key Words: Fractional Brownian motion; intersection local time; $k$-th derivative of intersection local time; exponential integrability.

Mathematics Subject Classifications (2010): 60G22; 60 J55

\section{Introduction and main result}

Intersection local time or self-intersection local time when the two processes are the same are important subjects in probability theory and their derivatives have received much attention recently. Jung et al. 2] and 3] discussed Tanaka formula and occupation-time formula for derivative self-intersection local time of fractional Brownian motions. On the other hand, several authors paid attention to the renormalized self-intersection local time of fractional Brownian motions, see e.g., Hu et al. [5] and [6].

Motivated by [2] and [4, higher-order derivative of intersection local time for two independent fractional Brownian motions is studied in this paper.

\footnotetext{
* Jingjun Guo acknowledges the support of National Natural Science Foundation of China \#71561017, the Youth Academic Talent Plan of Lanzhou University of Finance and Economics. Yaozhong Hu is partially supported by a grant from the Simons Foundation \#209206 and by a General Research Fund of University of Kansas. Yanping Xiao acknowledges the support of Northwest University for Nationalities \#xbmuyjrc201701.

†Corresponding author. gjjemail@126.com
} 
To state our main result we let $B^{H_{1}}=\left\{B_{t}^{H_{1}}, t \geq 0\right\}$ and $\widetilde{B}^{H_{2}}=\left\{\widetilde{B}_{t}^{H_{2}}, t \geq\right.$ $0\}$ be two independent $d$-dimensional fractional Brownian motions of Hurst parameters $H_{1}, H_{2} \in(0,1)$ respectively. This means that $B^{H_{1}}$ and $\widetilde{B}^{H_{2}}$ are independent centered Gaussian processes with covariance

$$
\mathbb{E}\left[B_{s}^{H_{1}} B_{t}^{H_{1}}\right]=\frac{1}{2}\left(s^{2 H_{1}}+t^{2 H_{1}}-|s-t|^{2 H_{1}}\right) .
$$

(similar identity for $\tilde{B})$.

In this paper we are concerned with the derivatives of intersection local time of $B^{H_{1}}$ and $\widetilde{B}^{H_{2}}$, defined by

$$
\hat{\alpha}^{(k)}(x):=\frac{\partial^{k}}{\partial x_{1}^{k_{1}} \cdots \partial x_{d}^{k_{d}}} \int_{0}^{T} \int_{0}^{T} \delta\left(B_{t}^{H_{1}}-\widetilde{B}_{s}^{H_{2}}+x\right) d t d s,
$$

where $k=\left(k_{1}, \cdots, k_{d}\right)$ is a multi-index with all $k_{i}$ being nonnegative integers and $\delta$ is the Dirac delta function of $d$-variable. In particular, we are exclusively consider the case when $x=0$ in this work. Namely, we are studying

$$
\hat{\alpha}^{(k)}(0):=\int_{0}^{T} \int_{0}^{T} \delta^{(k)}\left(B_{t}^{H_{1}}-\widetilde{B}_{s}^{H_{2}}\right) d t d s,
$$

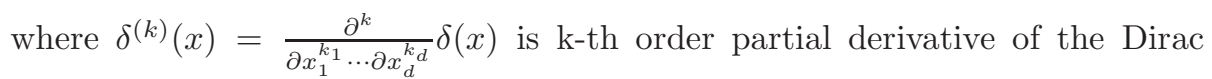
delta function. Since $\delta(x)=0$ when $x \neq 0$ the intersection local time $\hat{\alpha}(0)$ (when $k=0$ ) measures the frequency that processes $B^{H_{1}}$ and $\widetilde{B}^{H_{2}}$ intersect each other.

Since the Dirac delta function $\delta$ is a generalized function, we need to give a meaning to $\hat{\alpha}^{(k)}(0)$. To this end, we approximate the Dirac delta function $\delta$ by

$$
f_{\varepsilon}(x):=\frac{1}{(2 \pi \varepsilon)^{\frac{d}{2}}} e^{-\frac{|x|^{2}}{2 \varepsilon}}=\frac{1}{(2 \pi)^{d}} \int_{\mathbb{R}^{d}} e^{i p x} e^{-\frac{\varepsilon|p|^{2}}{2}} d p,
$$

and throughout this paper, we use $p x=\sum_{j=1}^{d} p_{j} x_{j}$ and $|p|^{2}=\sum_{j=1}^{d} p_{j}^{2}$. Thus we approximate $\delta^{(k)}$ by

$$
f_{\varepsilon}^{(k)}(x):=\frac{\partial^{k}}{\partial x_{1}^{k_{1}} \cdots \partial x_{d}^{k_{d}}} f_{\varepsilon}(x)=\frac{i^{k}}{(2 \pi)^{d}} \int_{\mathbb{R}^{d}} p_{1}^{k_{1}} \cdots p_{d}^{k_{d}} e^{i p x} e^{-\frac{\varepsilon|p|^{2}}{2}} d p .
$$

We say that $\hat{\alpha}^{(k)}(0)$ exists (in $\left.L^{2}\right)$ if

$$
\hat{\alpha}_{\varepsilon}^{(k)}(0):=\int_{0}^{T} \int_{0}^{T} f_{\varepsilon}^{(k)}\left(B_{t}^{H_{1}}-\widetilde{B}_{s}^{H_{2}}\right) d t d s
$$

converges to a random variable (denoted by $\hat{\alpha}^{(k)}(0)$ ) in $L^{2}$ when $\varepsilon \downarrow 0$.

Here is the main result of this work.

Theorem 1. Let $B^{H_{1}}$ and $\widetilde{B}^{H_{2}}$ be two independent d-dimensional fractional Brownian motions of Hurst parameter $\mathrm{H}_{1}$ and $\mathrm{H}_{2}$, respectively. 
(i) Assume $k=\left(k_{1}, \cdots, k_{d}\right)$ is an index of nonnegative integers (meaning that $k_{1}, \cdots, k_{d}$ are nonnegative integers) satisfying

$$
\frac{H_{1} H_{2}}{H_{1}+H_{2}}(|k|+d)<1,
$$

where $|k|=k_{1}+\cdots+k_{d}$. Then, the $k$-th order derivative intersection local time $\hat{\alpha}^{(k)}(0)$ exists in $L^{p}(\Omega)$ for any $p \in[1, \infty)$.

(ii) Assume condition (5) is satisfied. There is a strictly positive constant $C_{d, k, T} \in(0, \infty)$ such that

$$
\mathbb{E}\left[\exp \left\{C_{d, k, T}\left|\widehat{\alpha}^{(k)}(0)\right|^{\beta}\right\}\right]<\infty,
$$

where $\beta=\frac{H_{1}+H_{2}}{2 d H_{1} H_{2}}$.

(iii) If $\hat{\alpha}^{(k)}(0) \in L^{1}(\Omega)$, where $k=\left(0, \cdots, 0, k_{i}, 0, \cdots, 0\right)$ with $k_{i}$ being even integer, then condition (5) must be satisfied.

Remark 1. (i) When $k=0$, we have that $\widehat{\alpha}^{(0)}(0)$ is in $L^{p}(\Omega)$ for any $p \in$ $[1, \infty)$ if $\frac{H_{1} H_{2}}{H_{1}+H_{2}} d<1$. In the special case $H_{1}=H_{2}=H$, this condition becomes $H d<2$, which is the condition obtained in Nualart et al. [8].

(ii) When $H_{1}=H_{2}=\frac{1}{2}$, we have the exponential integrability exponent $\beta=$

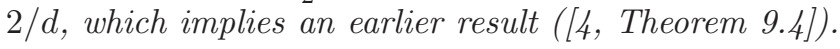

(iii) We also show that condition (5) is necessary in some sense. This is also first time.

\section{Proof of the theorem}

Proof of Parts (i) and (ii). This section is devoted to the proof of the theorem. We shall first find a good bound for $\mathbb{E}\left|\widehat{\alpha}^{(k)}(0)\right|^{n}$ which gives a proof for (i) and (ii) simultaneously. We introduce the following notations.

$$
\begin{aligned}
& p_{j}=\left(p_{1 j}, \ldots, p_{d j}\right), \quad p_{j}^{k}=\left(p_{1 j}^{k_{1}}, \ldots, p_{d j}^{k_{d}}\right), \quad j=1,2, \cdots, n ; \\
& p=\left(p_{1}, \ldots, p_{n}\right), \quad d p=\prod_{i=1}^{d} \prod_{j=1}^{n} d p_{i j} .
\end{aligned}
$$

We also denote $d s=d s_{1} \cdots d s_{n}$ and $d t=d t_{1} \cdots d t_{n}$. 
Fix an integer $n \geq 1$. Denote $T_{n}=\{0<t, s<T\}^{n}$. We have

$$
\begin{aligned}
\mathbb{E} & {\left[\left|\widehat{\alpha}_{\varepsilon}^{(k)}(0)\right|^{n}\right] } \\
\leq & \frac{1}{(2 \pi)^{n d}} \int_{T_{n}} \int_{\mathbb{R}^{n d}} \mid \mathbb{E}\left[\operatorname { e x p } \left\{i p_{1}\left(B_{s_{1}}^{H_{1}}-\widetilde{B}_{t_{1}}^{H_{2}}\right)+\cdots\right.\right. \\
& \left.\left.+i p_{n}\left(B_{s_{n}}^{H_{1}}-\widetilde{B}_{t_{n}}^{H_{2}}\right)\right\}\right]\left|\exp \left\{-\frac{\varepsilon}{2} \sum_{j=1}^{n}\left|p_{j}\right|^{2}\right\} \prod_{j=1}^{n}\right| p_{j}^{k} \mid d p d t d s \\
= & \frac{1}{(2 \pi)^{n d}} \int_{T_{n}} \int_{\mathbb{R}^{n d}} \exp \left\{-\frac{1}{2} \mathbb{E}\left[\sum_{j=1}^{n} p_{j}\left(B_{s_{j}}^{H_{1}}-\widetilde{B}_{t_{j}}^{H_{2}}\right)\right]^{2}\right\} \\
& \times \exp \left\{-\frac{\varepsilon}{2} \sum_{j=1}^{n}\left|p_{j}\right|^{2}\right\} \prod_{j=1}^{n}\left|p_{j}^{k}\right| d p d t d s \\
\leq & \frac{1}{(2 \pi)^{n d}} \int_{T_{n}} \int_{\mathbb{R}^{n d}} \prod_{i=1}^{d}\left(\prod_{j=1}^{n}\left|p_{i j}^{k_{i}}\right|\right) \exp \left\{-\frac{1}{2} \mathbb{E}\left[p_{i 1} B_{s_{1}}^{H_{1}, i}+\cdots+p_{i n} B_{s_{n}}^{H_{1}, i}\right]^{2}\right. \\
& \left.-\frac{1}{2} \mathbb{E}\left[p_{i 1} B_{t_{1}}^{H_{2}, i}+\cdots+p_{i n} B_{t_{n}}^{H_{2}, i}\right]^{2}\right\} d p d t d s .
\end{aligned}
$$

The expectations in the above exponent can be computed by

$$
\begin{aligned}
& \mathbb{E}\left[p_{i 1} B_{s_{1}}^{H_{1}, i}+\cdots+p_{i n} B_{s_{n}}^{H_{1}, i}\right]^{2}=\left(p_{i 1}, \cdots, p_{i n}\right) Q_{1}\left(p_{i 1}, \cdots, p_{i n}\right)^{T}, \\
& \mathbb{E}\left[p_{i 1} \tilde{B}_{s_{1}}^{H_{2}, i}+\cdots+p_{i n} \tilde{B}_{s_{n}}^{H_{2}, i}\right]^{2}=\left(p_{i 1}, \cdots, p_{i n}\right) Q_{2}\left(p_{i 1}, \cdots, p_{i n}\right)^{T},
\end{aligned}
$$

where

$$
Q_{1}=\mathbb{E}\left(B_{j}^{H_{1}, i} B_{k}^{H_{1}, i}\right)_{1 \leq j, k \leq n} \quad \text { and } \quad Q_{2}=\mathbb{E}\left(\tilde{B}_{j}^{H_{2}, i} \tilde{B}_{k}^{H_{2}, i}\right)_{1 \leq j, k \leq n}
$$

denote respectively covariance matrices of n-dimensional random vectors $\left(B_{s_{1}}^{H_{1}, i}, \ldots, B_{s_{n}}^{H_{1}, i}\right)$ and that of $\left(\widetilde{B}_{t_{1}}^{H_{2}, i}, \ldots, \widetilde{B}_{t_{n}}^{H_{2}, i}\right)$. Thus we have

$$
\mathbb{E}\left[\left|\widehat{\alpha}_{\varepsilon}^{(k)}(0)\right|^{n}\right] \leq \frac{1}{(2 \pi)^{n d}} \int_{T_{n}} \prod_{i=1}^{d} I_{i}(t, s) d t d s
$$

where

$$
I_{i}(t, s):=\int_{\mathbb{R}^{n}}\left|x^{k_{i}}\right| \exp \left\{-\frac{1}{2} x^{T}\left(Q_{1}+Q_{2}\right) x\right\} d x .
$$

Here we recall $x=\left(x_{1}, \cdots, x_{n}\right)$ and $x_{i}^{k}=x_{1}^{k_{i}} \cdots x_{n}^{k_{i}}$.

For each fixed $i$ let us compute integral $I_{i}(t, s)$ first. Denote $B=Q_{1}+Q_{2}$. Then $B$ is a strictly positive definite matrix and hence $\sqrt{B}$ exists. Making substitution $\xi=\sqrt{B} x$. Then

$$
I_{i}(t, s)=\int_{\mathbb{R}^{n}} \prod_{j=1}^{n}\left|\left(B^{-\frac{1}{2}} \xi\right)_{j}\right|^{k_{i}} \exp \left\{-\frac{1}{2}|\xi|^{2}\right\} \operatorname{det}(B)^{-\frac{1}{2}} d \xi .
$$


To obtain a nice bound for the above integral, let us first diagonalize $B$ :

$$
B=Q \Lambda Q^{-1},
$$

where $\Lambda=\operatorname{diag}\left\{\lambda_{1}, \ldots, \lambda_{n}\right\}$ is a strictly positive diagonal matrix with $\lambda_{1} \leq \lambda_{2} \leq$ $\cdots \leq \lambda_{d}$ and $Q=\left(q_{i j}\right)_{1 \leq i, j \leq d}$ is an orthogonal matrix. Hence, we have $\operatorname{det}(B)=$ $\lambda_{1} \cdots \lambda_{d}$. Denote

$$
\eta=\left(\eta_{1}, \eta_{2}, \cdots, \eta_{n}\right)^{T}=Q^{-1} \xi,
$$

Hence,

$$
\begin{aligned}
B^{-\frac{1}{2}} \xi= & Q \Lambda^{-1 / 2} Q^{-1} \xi=Q \Lambda^{-1 / 2} \eta \\
& =Q\left(\begin{array}{c}
\lambda_{1}^{-\frac{1}{2}} \eta_{1} \\
\lambda_{2}^{-\frac{1}{2}} \eta_{2} \\
\vdots \\
\lambda_{n}^{-\frac{1}{2}} \eta_{n}
\end{array}\right)=\left(\begin{array}{cccc}
q_{1,1} & q_{1,2} & \cdots & q_{1, n} \\
q_{2,1} & q_{2,2} & \cdots & q_{2, n} \\
\vdots & \vdots & \cdots & \vdots \\
q_{n, 1} & q_{n, 2} & \cdots & q_{n, n}
\end{array}\right)\left(\begin{array}{c}
\lambda_{1}^{-\frac{1}{2}} \eta_{1} \\
\lambda_{2}^{-\frac{1}{2}} \eta_{2} \\
\vdots \\
\lambda_{n}^{-\frac{1}{2}} \eta_{n}
\end{array}\right) .
\end{aligned}
$$

Therefore, we have

$$
\begin{aligned}
\left|\left(B^{-\frac{1}{2}} \xi\right)_{j}\right| & =\left|\sum_{k=1}^{n} q_{j k} \lambda_{k}^{-\frac{1}{2}} \eta_{k}\right| \leq \lambda_{1}^{-\frac{1}{2}} \sum_{k=1}^{n}\left|q_{j k} \eta_{k}\right| \\
& \leq \lambda_{1}^{-\frac{1}{2}}\left(\sum_{k=1}^{n} q_{j k}^{2}\right)^{\frac{1}{2}}\left(\sum_{k=1}^{n} \eta_{k}^{2}\right)^{\frac{1}{2}} \leq \lambda_{1}^{-\frac{1}{2}}|\eta|_{2}=\lambda_{1}^{-\frac{1}{2}}|\xi|_{2} .
\end{aligned}
$$

Since both $Q_{1}$ and $Q_{2}$ are positive definite, we see that

$$
\lambda_{1} \geq \lambda_{1}\left(Q_{1}\right), \quad \text { and } \quad \lambda_{1} \geq \lambda_{1}\left(Q_{2}\right),
$$

where $\lambda_{1}\left(Q_{i}\right)$ is the smallest eigenvalue of $Q_{i}, i=1,2$. This means that

$$
\lambda_{1} \geq \lambda_{1}\left(Q_{1}\right)^{\rho} \lambda_{1}\left(Q_{2}\right)^{1-\rho} \quad \text { for any } \rho \in[0,1] .
$$

This implies

$$
\left|\left(B^{-\frac{1}{2}} \xi\right)_{j}\right| \leq \lambda_{1}\left(Q_{1}\right)^{-\frac{1}{2} \rho} \lambda_{1}\left(Q_{2}\right)^{-\frac{1}{2}(1-\rho)}|\xi|_{2} .
$$

Consequently, we have

$$
\begin{gathered}
I_{i}(t, s)=\operatorname{det}(B)^{-\frac{1}{2}} \lambda_{1}\left(Q_{1}\right)^{-\frac{1}{2} \rho k_{i}} \lambda_{1}\left(Q_{2}\right)^{-\frac{1}{2}(1-\rho) k_{i}} \\
\int_{\mathbb{R}^{n}}|\xi|_{2}^{k_{i}} \exp \left\{-\frac{1}{2}|\xi|^{2}\right\} d \xi,
\end{gathered}
$$

for any $\rho \in[0,1]$.

Now we are going to find a lower bound for $\lambda_{1}\left(Q_{1}\right)\left(\lambda_{1}\left(Q_{2}\right)\right.$ can be dealt with the same way. We only need to replace $s$ by $t)$. Without loss of generality 
we can assume $0 \leq s_{1}<s_{2}<\cdots<s_{n} \leq T$. From the definition of $Q_{1}$ we have for any vector $u=\left(u_{1}, \cdots, u_{d}\right)^{T}$,

$$
\begin{aligned}
& u^{T} Q_{1} u=\operatorname{Var}\left(u_{1} B_{s_{1}}^{H_{1}}+u_{2} B_{s_{2}}^{H_{1}}+\ldots+u_{n} B_{s_{n}}^{H_{1}}\right) \\
& \quad=\operatorname{Var}\left(\left(u_{1}+\ldots+u_{n}\right) B_{s_{1}}^{H_{1}}+\left(u_{2}+\ldots+u_{n}\right)\left(B_{s_{2}}^{H_{1}}-B_{s_{1}}^{H_{1}}\right)\right. \\
& \left.\quad+\ldots+\left(u_{n-1}+u_{n}\right)\left(B_{s_{n-1}}^{H_{1}}-B_{s_{n-2}}^{H_{1}}\right)+u_{n}\left(B_{s_{n}}^{H_{1}}-B_{s_{n-1}}^{H_{1}}\right)\right)
\end{aligned}
$$

Now using Lemma 8.1 of 1 and Lemma A.1 of [6], we see that $R$ appeared in Lemma 8.1 satisfies $R \geq \prod_{i=1}^{n} \sigma_{i}^{2}$ ( $m$ in Lemma 8.1 of [1] is replaced by $n$ ). Thus we have

$$
\begin{aligned}
u^{T} Q_{1} u & \geq\left(\left(u_{1}+\ldots+u_{n}\right)^{2} s_{1}^{2 H_{1}}+\left(u_{2}+\ldots+u_{n}\right)^{2}\left(s_{2}-s_{1}\right)^{2 H_{1}}\right. \\
& \left.+\ldots+\left(u_{n-1}+u_{n}\right)^{2}\left(s_{n-1}-s_{n-2}\right)^{2 H_{1}}+u_{n}^{2}\left(s_{n}-s_{n-1}\right)^{2 H_{1}}\right) \\
& \geq \min \left\{s_{1}^{2 H_{1}},\left(s_{2}-s_{1}\right)^{2 H_{1}}, \ldots,\left(s_{n}-s_{n-1}\right)^{2 H_{1}}\right\} \\
& {\left[\left(u_{1}+\ldots+u_{n}\right)^{2}+\left(u_{2}+\ldots+u_{n}\right)^{2}+\ldots+\left(u_{n-1}+u_{n}\right)^{2}+u_{n}^{2}\right] . }
\end{aligned}
$$

Consider the function

$$
\begin{aligned}
f\left(u_{1}, \cdots, u_{n}\right) & =\left(u_{1}+\cdots+u_{n}\right)^{2}+\left(u_{2}+\cdots+u_{n}\right)^{2}+\cdots+\left(u_{n-1}+u_{n}\right)^{2}+u_{n}^{2} \\
& =\left(u_{1}, \cdots, u_{n}\right) G\left(u_{1}, \cdots, u_{n}\right)^{T}
\end{aligned}
$$

where

$$
G=\left(\begin{array}{ccccc}
1 & 1 & 1 & \ldots & 1 \\
0 & 1 & 1 & \ldots & 1 \\
\vdots & \vdots & \vdots & \ldots & \vdots \\
0 & 0 & 0 & \ldots & 1
\end{array}\right)
$$

It is easy to see that the matrix $G^{T} G$ has a minimum eigenvalue independent of $n$. Thus this function $f$ attains its minimum value $f_{\min }$ independent of $n$ on the sphere $u_{1}^{2}+\cdots+u_{n}^{2}=1$. It is also easy to see that $f_{\min }>0$.

As a consequence we have

$$
\begin{aligned}
& \lambda_{1}\left(Q_{1}\right)=\inf _{|u|=1} u^{T} Q_{1} u \\
& \quad \geq \min \left\{s_{1}^{2 H_{1}},\left(s_{2}-s_{1}\right)^{2 H_{1}}, \ldots,\left(s_{n}-s_{n-1}\right)^{2 H_{1}}\right\} \inf _{|u|=1} f\left(u_{1}, \cdots, u_{n}\right) \\
& \quad \geq f_{\min } \min \left\{s_{1}^{2 H_{1}},\left(s_{2}-s_{1}\right)^{2 H_{1}}, \ldots,\left(s_{n}-s_{n-1}\right)^{2 H_{1}}\right\} \\
& \quad \geq K \min \left\{s_{1}^{2 H_{1}},\left(s_{2}-s_{1}\right)^{2 H_{1}}, \ldots,\left(s_{n}-s_{n-1}\right)^{2 H_{1}}\right\}
\end{aligned}
$$

In a similar way we have

$$
\lambda_{1}\left(Q_{2}\right) \geq K \min \left\{t_{1}^{2 H_{2}},\left(t_{2}-t_{1}\right)^{2 H_{2}}, \ldots,\left(t_{n}-t_{n-1}\right)^{2 H_{2}}\right\} .
$$


The integral in (7) can be bounded as

$$
\begin{aligned}
I_{2} & :=\int_{\mathbb{R}^{n}}|\xi|^{k_{i}} \exp \left\{-\frac{1}{2}|\xi|^{2}\right\} d \xi \\
& \leq n^{\frac{k_{i}}{2}} \int_{\mathbb{R}^{n d}} \max _{1 \leq j \leq n}\left|\xi_{j}\right|^{k_{i}} \exp \left\{-\frac{1}{2}|\xi|^{2}\right\} d \xi \\
& \leq n^{\frac{k_{i}}{2}} \int_{\mathbb{R}^{n}} \sum_{j=1}^{n}\left|\xi_{j}\right|^{k_{j}} \exp \left\{-\frac{1}{2}|\xi|^{2}\right\} d \xi \\
& \leq n^{\frac{k_{i}}{2}+1} \int_{\mathbb{R}^{n}}\left|\xi_{1}\right|^{k_{i}} \exp \left\{-\frac{1}{2}|\xi|^{2}\right\} d \xi \\
& \leq n^{\frac{k_{i}}{2}+1} C^{n} \leq C^{n} .
\end{aligned}
$$

Substitute (8)-(10) into (7) we obtain

$$
\begin{gathered}
I_{i}(t, s) \leq C^{n} \operatorname{det}(B)^{-\frac{1}{2}} \min _{j=1, \ldots, n}\left(s_{j}-s_{j-1}\right)^{-\rho H_{1} k_{i}} \\
\min _{j=1, \ldots, n}\left(t_{j}-t_{j-1}\right)^{-(1-\rho) H_{2} k_{i}} .
\end{gathered}
$$

Next we obtain a lower bound for $\operatorname{det}(B)$. According to [4, Lemma 9.4]

$$
\operatorname{det}\left(Q_{1}+Q_{2}\right) \geq \operatorname{det}\left(Q_{1}\right)^{\gamma} \operatorname{det}\left(Q_{2}\right)^{1-\gamma},
$$

for any two symmetric positive definite matrices $Q_{1}$ and $Q_{2}$ and for any $\gamma \in$ $[0,1]$. Now it is well-known that (see also the usages in [4], [5] and [6]).

$$
\operatorname{det}\left(Q_{1}\right) \geq C^{n} s_{1}^{2 H_{1}}\left(s_{2}-s_{1}\right)^{2 H_{1}} \cdots\left(s_{n}-s_{n-1}\right)^{2 H_{1}} .
$$

and

$$
\operatorname{det}\left(Q_{2}\right) \geq C^{n} t_{1}^{2 H_{2}}\left(t_{2}-t_{1}\right)^{2 H_{2}} \cdots\left(t_{n}-t_{n-1}\right)^{2 H_{2}} .
$$

As a consequence, we have

$$
\begin{aligned}
I_{i}(t, s) \leq C^{n} \min _{j=1, \ldots, n}\left(s_{j}-s_{j-1}\right)^{-\rho H_{1} k_{i}} \min _{j=1, \ldots, n}\left(t_{j}-t_{j-1}\right)^{-(1-\rho) H_{2} k_{i}} \\
\\
\quad\left[s_{1}\left(s_{2}-s_{1}\right) \cdots\left(s_{n}-s_{n-1}\right)\right]^{-\gamma H_{1}}\left[t_{1}\left(t_{2}-t_{1}\right) \cdots\left(t_{n}-t_{n-1}\right)\right]^{-(1-\gamma) H_{2}} .
\end{aligned}
$$

Thus,

$$
\begin{aligned}
\mathbb{E}\left[\left|\widehat{\alpha}_{\varepsilon}^{(k)}(0)\right|^{n}\right] \leq(n !)^{2} C^{n} \int_{\Delta_{n}^{2}} \min _{j=1, \ldots, n}\left(s_{j}-s_{j-1}\right)^{-\rho H_{1}|k|} \\
\min _{j=1, \ldots, n}\left(t_{j}-t_{j-1}\right)^{-(1-\rho) H_{2}|k|}\left[s_{1}\left(s_{2}-s_{1}\right) \cdots\left(s_{n}-s_{n-1}\right)\right]^{-\gamma H_{1} d} \\
\quad\left[t_{1}\left(t_{2}-t_{1}\right) \cdots\left(t_{n}-t_{n-1}\right)\right]^{-(1-\gamma) H_{2} d} d t d s \\
\leq(n !)^{2} C^{n} \sum_{i, j=1}^{n} \int_{\Delta_{n}^{2}}\left(s_{i}-s_{i-1}\right)^{-\rho H_{1}|k|} \\
\left(t_{j}-t_{j-1}\right)^{-(1-\rho) H_{2}|k|}\left[s_{1}\left(s_{2}-s_{1}\right) \cdots\left(s_{n}-s_{n-1}\right)\right]^{-\gamma H_{1} d} \\
{\left[t_{1}\left(t_{2}-t_{1}\right) \cdots\left(t_{n}-t_{n-1}\right)\right]^{-(1-\gamma) H_{2} d} d t d s, }
\end{aligned}
$$


where $\Delta_{n}=\left\{0<s_{1}<\cdots<s_{n} \leq T\right\}$ denotes the simplex in $[0, T]^{n}$. We choose $\rho=\gamma=\frac{H_{2}}{H_{1}+H_{2}}$ to obtain

$$
\mathbb{E}\left[\left|\widehat{\alpha}_{\varepsilon}^{(k)}(0)\right|^{n}\right] \leq(n !)^{2} C^{n} \sum_{i, j=1}^{n} I_{3, i} I_{3, j}
$$

where

$$
I_{3, j}=\int_{\Delta_{n}}\left(t_{j}-t_{j-1}\right)^{-\frac{H_{1} H_{2}}{H_{1}+H_{2}}|k|}\left[t_{1}\left(t_{2}-t_{1}\right) \cdots\left(t_{n}-t_{n-1}\right)\right]^{-\frac{H_{1} H_{2}}{H_{1}+H_{2}} d} d t,
$$

By Lemma 4.5 of [7, we see that if

$$
\frac{H_{1} H_{2}}{H_{1}+H_{2}}(|k|+d) \leq 1,
$$

then

$$
I_{3, j} \leq \frac{C^{n} T^{\kappa_{1} n-\frac{H_{1} H_{2}|k|}{H_{1}+H_{2}}}}{\Gamma\left(n \kappa_{1}-\frac{H_{1} H_{2}}{H_{1}+H_{2}}|k|+1\right)},
$$

where

$$
\kappa_{1}=1-\frac{d H_{1} H_{2}}{H_{1}+H_{2}} .
$$

Substituting this bound we obtain

$$
\begin{aligned}
& \mathbb{E}\left[\left|\widehat{\alpha}_{\varepsilon}^{(k)}(0)\right|^{n}\right] \leq n^{2}(n !)^{2} C^{n} \frac{T^{2 \kappa_{1} n-\frac{2 H_{1} H_{2}|k|}{H_{1}+H_{2}}}}{\Gamma^{2}\left(n \kappa_{1}-\frac{H_{1} H_{2}}{H_{1}+H_{2}}|k|+1\right)} \\
& \leq(n !)^{2} C^{n} \frac{T^{2 \kappa_{1} n-\frac{2 H_{1} H_{2}|k|}{H_{1}+H_{2}}}}{\left(\Gamma\left(n \kappa_{1}-\frac{H_{1} H_{2}}{H_{1}+H_{2}}|k|+1\right)\right)^{2}} \\
& \leq C_{T}(n !)^{2-2 \kappa_{1}} C^{n} T^{2 \kappa_{1} n}
\end{aligned}
$$

where $C$ is a constant independent of $T$ and $n$ and $C_{T}$ is a constant independent of $n$.

For any $\beta>0$, the above inequality implies

$$
\mathbb{E}\left[\left|\widehat{\alpha}^{(k)}(0)\right|^{n \beta}\right] \leq C_{T}(n !)^{\beta\left(2-2 \kappa_{1}\right)} C^{n} T^{2 \beta \kappa_{1} n}
$$

From this bound we conclude that there exists a constant $C_{d, T, k}>0$ such that

$$
\begin{gathered}
\mathbb{E}\left[\exp \left\{C_{d, T, k}\left|\widehat{\alpha}^{(k)}(0)\right|^{\beta}\right\}\right]=\sum_{n=0}^{\infty} \frac{C_{d, T, k}^{n}}{n !} \mathbb{E}\left|\widehat{\alpha}^{(k)}(0)\right|^{n \beta} \\
\leq C_{T} \sum_{n=0}^{\infty} C_{d, T, k}^{n}(n !)^{\beta\left(2-2 \kappa_{1}\right)-1} C^{n} T^{2 \beta \kappa_{1} n}<\infty
\end{gathered}
$$


when $C_{d, T, k}$ is sufficiently small (but strictly positive), where $\beta=\frac{H_{1}+H_{2}}{2 d H_{1} H_{2}}$.

Proof of part (iii). Without loss generality, we consider only the case $k=$ $\left(k_{1}, 0, \cdots, 0\right)$ and we denote $k_{i}$ by $k$. By the definition of k-order derivative local time of independent d-dimensional fractional Brownian motions, we have

$$
\begin{aligned}
\mathbb{E}\left[\hat{\alpha}_{\varepsilon}^{(k)}(0)\right] & =\frac{1}{(2 \pi)^{d}} \int_{0}^{T} \int_{0}^{T} \int_{\mathbb{R}^{d}} \mathbb{E}\left[e^{i\left\langle\xi, B_{t}^{H_{1}}-\widetilde{B}_{s}^{H_{2}}\right\rangle}\right] e^{-\frac{\varepsilon|\xi|^{2}}{2}}\left|\xi_{1}\right|^{k} d \xi d t d s \\
& =\frac{1}{(2 \pi)^{d}} \int_{0}^{T} \int_{0}^{T} \int_{\mathbb{R}^{d}} e^{-\left(\varepsilon+t^{2 H_{1}}+s^{2 H_{2}}\right) \frac{|\xi|^{2}}{2}}\left|\xi_{1}\right|^{k} d \xi d t d s .
\end{aligned}
$$

Thus, we have

$$
\mathbb{E}\left[\hat{\alpha}^{(k)}(0)\right]=\frac{1}{(2 \pi)^{d}} \int_{0}^{T} \int_{0}^{T} \int_{\mathbb{R}^{d}} e^{-\left(t^{2 H_{1}}+s^{2 H_{2}}\right) \frac{|\xi|^{2}}{2}}\left|\xi_{1}\right|^{k} d \xi d t d s .
$$

Integrating with respect to $\xi$, we find

$$
\mathbb{E}\left[\hat{\alpha}^{(k)}(0)\right]=c_{k, d} \int_{0}^{T} \int_{0}^{T}\left(t^{2 H_{1}}+s^{2 H_{2}}\right)^{-\frac{(k+d)}{2}} d t d s
$$

for some constant $c_{k, d} \in(0, \infty)$.

We are going to deal with the above integral. Assume first $0<H_{1} \leq H_{2}<1$. Making substitution $t=u^{\frac{H_{2}}{H_{1}}}$ yields

$$
\begin{aligned}
I_{4} & :=\int_{0}^{T} \int_{0}^{T}\left(t^{2 H_{1}}+s^{2 H_{2}}\right)^{-\frac{(k+d)}{2}} d t d s \\
& =\int_{0}^{T} \int_{0}^{T^{\frac{H_{1}}{H_{2}}}}\left(u^{2 H_{2}}+s^{2 H_{2}}\right)^{-\frac{k+d}{2}} u^{\frac{H_{2}}{H_{1}}-1} d u d s .
\end{aligned}
$$

Using polar coordinate $u=r \cos \theta$ and $s=r \sin \theta$, where $0 \leq \theta \leq \frac{\pi}{2}$ and $0 \leq r \leq T$ we have

$$
I_{4} \geq \int_{0}^{\frac{\pi}{2}}(\cos \theta)^{\frac{H_{2}}{H_{1}}-1}\left(\cos ^{2 H_{2}} \theta+\sin ^{2 H_{2}} \theta\right)^{-\frac{(k+d)}{2}} d \theta \int_{0}^{T^{\frac{H_{1}}{H_{2}}}} r^{-(k+d) H_{2}+\frac{H_{2}}{H_{1}}} d r
$$

since the planar domain $\left\{(r, \theta), 0 \leq r \leq T \wedge T^{\frac{H_{1}}{H_{2}}}, 0 \leq \theta \leq \frac{\pi}{2}\right\}$ is contained in the planar domain $\left\{(s, u), 0 \leq s \leq T, 0 \leq u \leq T^{\frac{H_{1}}{H_{2}}}\right\}$. The integral with respect to $r$ appearing in (12) is finite only if $-(k+d) H_{2}+\frac{H_{2}}{H_{1}}>-1$, namely, only when the condition (5) is satisfied. The case $0<H_{2} \leq H_{1}<1$ can be dealt similarly. This completes the proof of our main theorem. 


\section{References}

[1] S. M. Berman. Local nondeterminism and local times of Gaussian processes. Indiana Univ. Math. J., 1973, 23: 69-94.

[2] P. Jung, G. Markowsky. On the Tanaka formula for the derivative of selfintersection local time of fractional Brownian motion. Stoc. Proces. Their Appl., 2014, 124: 3846-3868.

[3] P. Jung, G. Markowsky. Hölder continuity and occupation-time formulas for $\mathrm{fBm}$ self-intersection local time and its derivative. J. Theor. Probab., 2015, 28: 299-312.

[4] Y. Hu. Analysis on Gaussian spaces. World Scientific, 2016.

[5] Y. Hu, D. Nualart. Renormalized self-intersection local time for fractional Brownian motion. Ann. Pro., 2005, 33(3): 948-983.

[6] Y. Hu, D. Nualart, J. Song. Integral representation of renormalized selfintersection local times. J. Func. Anal., 2008, 255(9): 2507-2532.

[7] Y. Hu, J. Huang, D. Nualart, S. Tindel. Stochastic heat equations with general multiplicative Gaussian noises: Hölder continuity and intermittency. Electron. J. Probab., 2015, 20(55): 1-50.

[8] D. Nualart, S. Ortiz-Latorre. Intersection local time for two independent fractional Brownian motions. J. Theor. Prob., 2007, 20: 759-767.

[9] M. Oliveira, J. Silva, L. Streit. Intersection local times of independent fractional Brownian motions as generalized white noise functionals. Acta Appl. Math., 2011, 113(1): 17-39.

[10] D. Wu, Y. Xiao. Regularity of intersection local times of fractional Brownian motions. J. Theor. Prob., 2010, 23(4): 972-1001.

[11] L. Yan. Derivative for the intersection local time of fractional Brownian motions. arXiv:1403.4102v3, 2014.

[12] L. Yan, X. Yu. Derivative for self-intersection local time of multidimensional fractional Brownian motion. Stoch., 2015, 87(6): 966-999. 